\title{
RECENT STUDIES ON BEAM-PHOTOELECTRON INSTABILITY IN BEPC
}

\author{
Z. Y. Guo, H. Huang, S. P. Li, D. K. Liu, Y. Luo, L. Ma, Q. Qin, L. F. Wang, J. Q. Wang, \\ S. H. Wang, J. W. Xu, K. R. Ye, C. Zhang, F. Zhou, IHEP, Beijing China \\ Y. H. Chin, H. Fukuma, S. Hiramatsu, M. Izawa, T. Kasuga, E. Kikutani, Y. Kobayashi, \\ S. Kurokawa, K. Ohmi, Y. Sato, Y. Suetsugu, M. Tobiyama, \\ K. Tsukamoto, K. Yokoya, KEK, Japan
}

\begin{abstract}
An experiment investigating the photoelectron instability has been performed in BEPC at IHEP, China. The betatron oscillation of each bunch was observed and recorded by a single pass beam position monitor system which has a capability to measure the transverse position of every bunch over 16000 turns. The effect of an octupole magnet on the instability was also studied. This paper describes results of those observations and analyses.
\end{abstract}

\section{INTRODUCTION}

Several years ago, an anomalous vertical coupled-bunch instability was observed[1] in PF at KEK, Japan. It occurs only with the positron beam and the threshold current is rather low. K. Ohmi proposed a physics model[2] which can qualitatively reproduce the phenomenon. According to this model, the instability is caused by the cloud of an enormous number of electrons which are produced by synchrotron light hitting the inner surface of the beam chamber. With reference to this mechanism, we call the instability, the Photo Electron Instability (PEI).

In order to investigate the nature of the PEI, a series of experiments have been carried out in BEPC since 1996 under the collaboration of IHEP, China and KEK, Japan. At the early stage of these experiments, we found an instability in BEPC, which was very similar to that observed in PF. After that we studied the dependence of the instability on several parameters such as chromaticity, bunch spacing, emittance, beam energy, RF frequency, on/off status of distributed ion bumps, betatron tunes, magnetic field, RF voltage and so forth. Results of these experiments were published in the Proceedings of PAC97[3], EPAC98[4] and APAC98[5].

The recent experiment was carried out in May 1998. Aims of this experiment were (1) measurement of vertical damping time, (2) time domain analysis of the oscillations with a single-pass beam position monitor (SPBPM) and (3) investigation the effect of an octupole field on the instability. In this paper, we report the results of these investigations.

\section{EXPERIMENTAL SETUP}

The main parameters in the experiment are given in Table1. Basic beam-diagnostics tools for the experiment are pickup electrode connected to a spectrum analyzer HP8568B and
Table 1: Main parameters in the experiment

$\begin{array}{ll}\text { Beam energy } & 1.3 \mathrm{GeV} \\ \text { Nominal betatron tune } & 5.82(\mathrm{H}) \text { and 6.74 (V) } \\ \text { Natural emittance } & 0.134 \mathrm{~mm} \mathrm{mr} \\ \text { RF frequency } & 200 \mathrm{MHz} \\ \text { Rad. damping time (trans.) } & 86 \mathrm{~ms} \\ \text { Harmonic number } & 160 \\ \text { Minimum bunch spacing } & 5 \mathrm{nsec}\end{array}$

a synchrotron light monitor, which is very useful to confirm the occurrence of the instability visually. Also a wall current monitor is indispensable for checking the relative population of particles in each RF bucket.

Another beam-diagnostic device, which was added this time, is the SPBPM developed at KEK[6]. This is a very powerful tool for investigating the nature of instabilities. Traditionally, the mode analysis of the instability has been done with a spectrum analyzer, which is essentially narrowband device. However, when the bunch spacing in the beam is very short, for instance, a few nano seconds, observing the spectrum around each revolution harmonic is very timeconsuming. Conditions of the instability may be changed during data acquisition due to a change of beam parameters, such as the beam current. The SPBPM system, in contrast to a spectrum analyzer, enables us to acquire data in a very short time, owing to its wide band-width. In addition, with this system we can study the time development of the instability.

The SPBPM system consists of (1) front-end electronics as a bunch position detector, (2) a fast A-to-D converter (FADC) and (3) a memory system which can store data up to $20 \mathrm{MB}$. A diagram of the FADC-memory system is shown in Fig. 1.

One set of the BPM electrodes are connected to the front-end electronics, and the position of every bunch was recorded turn by turn. Out of $20 \mathrm{MB}$ memory capacity, only 2.6 MB was used in each file. This corresponds to $16384\left(2^{14}\right)$ turns of 160 bunches (full bucket filling). The number of the turns 16384 was supposed to be large enough for the analysis of the phenomenon and was reasonable for quick semi-real-time analysis.

Another newly installed device is an octupole magnet to control the damping time of beam oscillation. Experience in PF showed that the octupole field introduces the damping to the instability. 


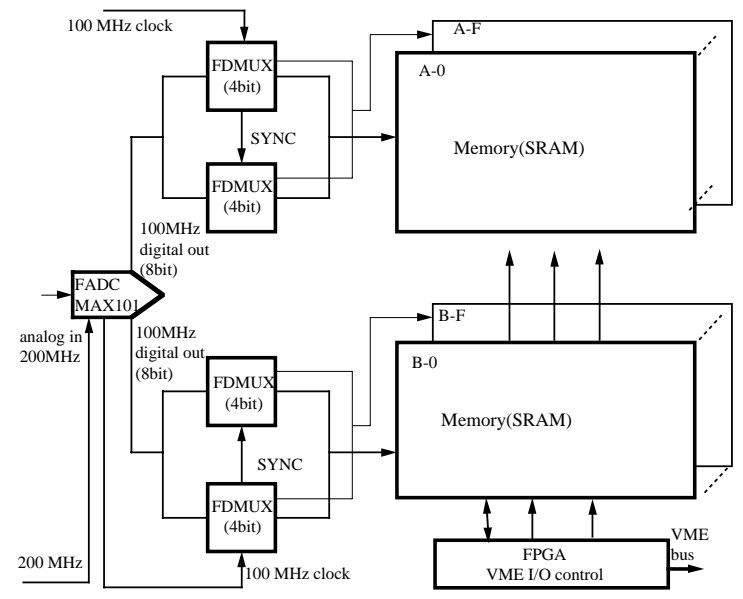

Figure 1: Block diagram of the FADC and the memory system.

\section{RESULTS AND DISCUSSIONS}

\subsection{Measurement of damping time in vertical plane}

The purpose of the damping time measurement is to estimate the growth time of the PEI at its threshold current. A single bunch with very low current which is at the level of the PEI threshold was stored in the ring, and betatron oscillation was resonantly excited by a stripline kicker. Just before the excitation is turned off, the data acquisition by the SPBPM system starts to record the damping behavior of the bunch.

The damping rate was obtained as follows. The 16384point data were divided into 64 sub-data each of which contains 256-point. Each sub-data was processed by Fourier transformation and amplitude corresponding to the tune was picked up. Then we have the amplitude as a function of time. When we fit this function with an exponentially damped function plus an offset, we get the damping rate.

The damping rate was measured for several values of the bunch current and the result is shown in Fig. 2. It shows us that the damping rate linearly increases as a function of bunch current. The line can be extrapolated to zero-current and is consistent with the radiation damping rate of $12 \mathrm{~s}^{-1}$. No Landau damping was observed in the experiment.

\subsection{Mode analysis}

A basic method of investigating coupled-bunch instabilities is the mode analysis, because the mode distribution holds the information on wake force which causes the instabilities. In this experiment we have done this mode analysis using the SPBPM data, which can track the behavior of the modes as a function of time.

The $160 \times 16384$-point data are divided into sub-data of $160 \times 256$-point data. The discrete Fourier transform was performed for each of the sub-data. The signal corresponding the tune (6.75) is picked up. Then we obtain mode spectra as a function of time.

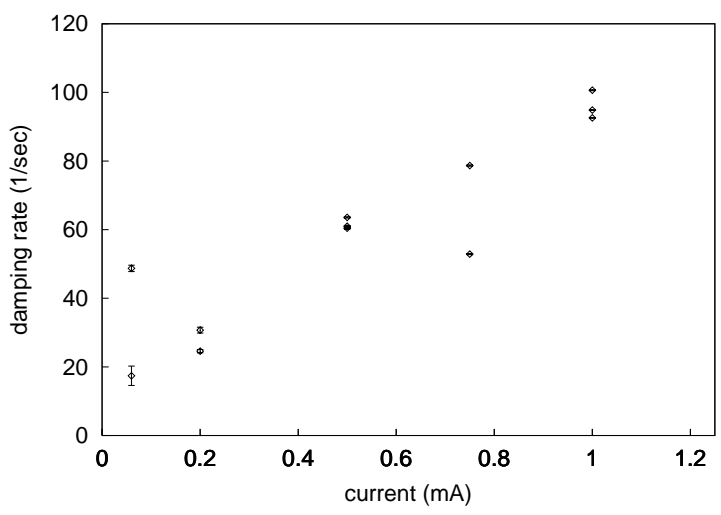

Figure 2: Damping rate as a function of bunch current.

Applying this method we analyzed a number of data taken for 160 positron bunches. One example of the time variation of mode amplitude is shown in Fig. 3. From this graph, we recognize two broad peaks within which many modes are growing and damping. It is a common characteristic of all the positron multi-bunch data. From the analysis results in this way, one can confirm the results obtained before with the spectrum analyzer[3].

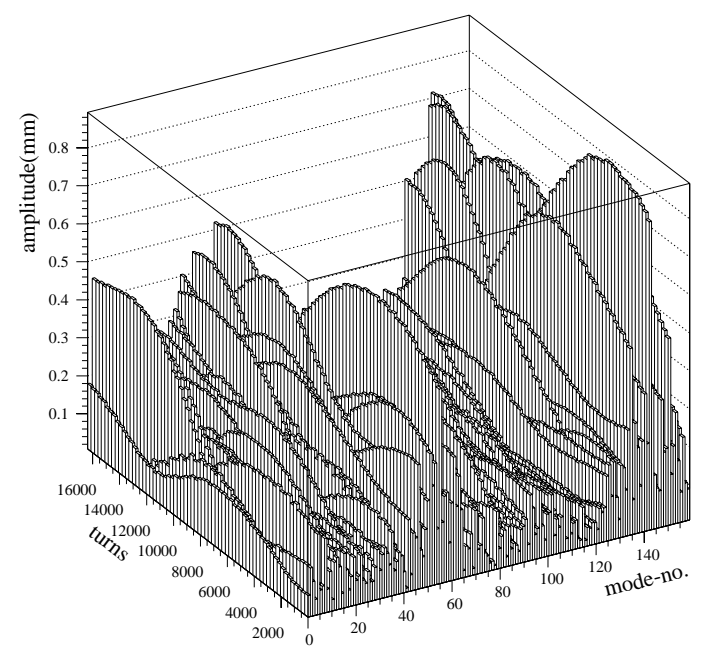

Figure 3: Behavior of the modes for positron bunches.

Two broad peaks in the mode distributions can be reproduced by a simple phenomenological model, with which the growth time $\tau_{s}$ is given by

$\tau_{s}^{-1}=\operatorname{Im}\left[A \sum_{p=1}^{l} F(-p D) \exp \left[2 p \pi i\left(m+\nu_{\beta}\right) / M\right]\right]$

where $A$ is a positive constant, $F$ a transverse wake which affects on the next $l$ bunches, $m$ the mode number, $\nu_{\beta}$ the betatron tune, $D$ the bunch spacing, and $M$ the bunch number. The growth rate in the mode distribution and position of the peaks of Fig. 4 are similar to that of the 
observation as shown in Fig. 3. This analysis shows us that the range of the wake is a few RF buckets. We adopted the rigid bunch simulation method in reference[4], in which the nonlinear wake force is involved, to track the behavior of the coherent coupled bunch oscillation. The simulation results show the same characteristic of the mode distribution as in Fig. 5.

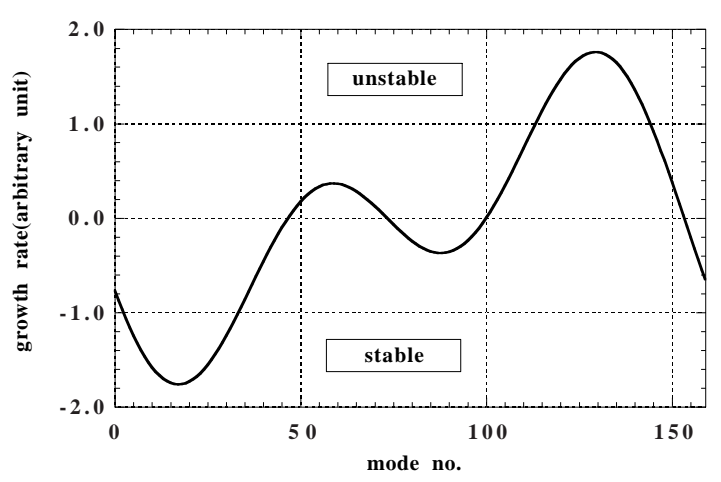

Figure 4: Growth rate calculated by a simple step-function wake.

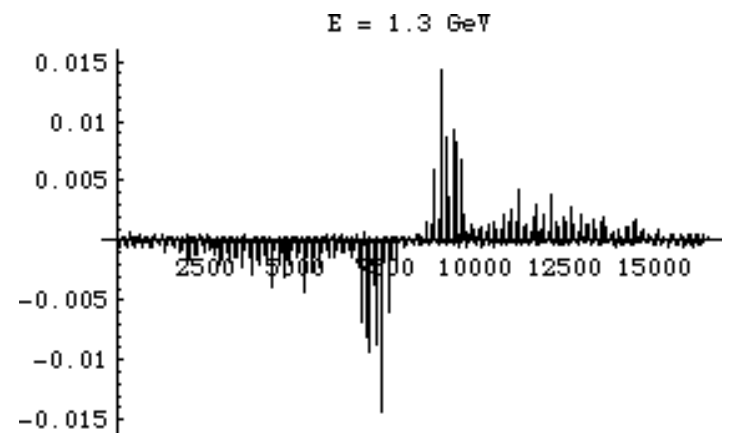

Figure 5: Mode distribution of the PEI

Fig. 6 depicts the time variation of the mode amplitude of electron beam which is totally different from that of the positron. There is one dominant mode whose mode number is 153. In the two beam model of the ion trapping[7], this mode can be excited if the ion tune $\nu_{i}$ is near $1-\delta \nu_{\beta}$, where $\delta \nu_{\beta}$ is a fractional tune. Under our experimental condition, the condition $1-\delta \nu_{\beta}$ is fulfilled if the beam current is $8.4 \mathrm{~mA}$, which is not so far from the beam current of $11.8 \mathrm{~mA}$ where data were taken.

\subsection{Effects of the octupole magnet}

An octupole magnet was installed in BEPC ring to observe its effect on the the instability. When the octupole was excited to $K_{3}=-33 \mathrm{~m}^{-3}$, the instability was suppressed. The Landau damping rate by the octupole was calculated by the canonical perturbation theory[8] and it shows the damping time of $6.5 \mathrm{~ms}$ which should be the estimate of the growth time of the instability. A simulation based on the PEI model gives the growth time of about $3 \mathrm{~ms}$ which is close to the estimated growth time due to the octupole effect observation.

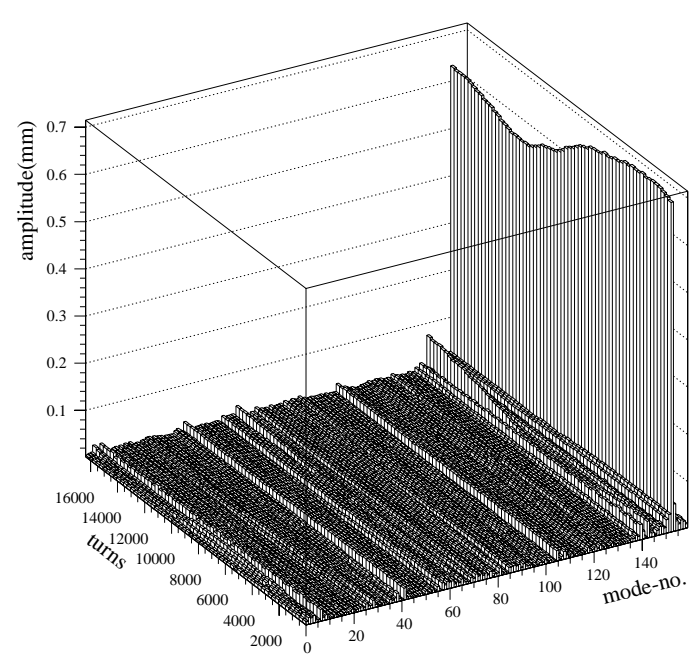

Figure 6: Behavior of modes for electron bunches.

\section{SUMMARY}

The photoelectron instability was studied in BEPC at IHEP, China. The SPBPM system was used to measure the bunch oscillation in the instability. Obtained mode spectrum shows two broad peaks. This has the same feature as the simulation based on the PEI model. The growth time of the instability was measured by exciting the octupole magnet. Estimated growth time by the measurement is $6.5 \mathrm{~ms}$ which is consistent with the result by the PEI simulation. The model of the beam-photoelectron instability is further confirmed by the recent studies on this phenomena in BEPC. No Landau was observed in the experiment. The reason why it was not observed is not understood yet.

\section{ACKNOWLEDGMENT}

The authors are grateful to the BEPC operation team. Discussions with M. Tigner are very helpful.

\section{REFERENCES}

[1] M. Izawa et al., Phys. Rev. Lett. 74, 5044 (1995).

[2] K. Ohmi, Phys. Rev. Lett. 75, 1526 (1995).

[3] Z.Y. Guo et al., Proceedings of 17th PAC, Vancouver, B.C., Canada, 1566(1997).

[4] Z. Y. Guo et al., Proceedings of EPAC98, Stockholm, Sweden, 957(1998).

[5] Z. Y. Guo et al. Proceedings of 1st APAC98, KEK, Tsukuba, Japan, 5d020(1998).

[6] E. Kikutani et al., Proceedings of EPAC96, Barcelona, Spain, 1893(1996).

[7] E. Keil and B. Zotter, CERN-ISR-TH/71-58(1971); D.G. Koshkarev, P.R.Zenkevich, Particle Accelerators 3,1(1972).

[8] K. Ohmi, KEK Report 97-9, IHEP/BEPC/AP/97-09. 